\title{
Extracellular synthesis of silver nanoparticles using dried leaves of pongamia pinnata $(L)$ pierre
}

Rajesh W. Raut1 ${ }^{1,}$, Niranjan S. Kolekar ${ }^{1}$, Jaya R. Lakkakula1, Vijay D. Mendhulkar ${ }^{1}$ and Sahebrao B. Kashid ${ }^{2}$

Extract of oven dried leaves of Pongamia pinnata (L) Pierre was used for the synthesis of silver nanoparticles. Stable and crystalline silver nanoparticles were formed by the treatment of aqueous solution of $\mathrm{AgNO}_{3}(1 \mathrm{mM})$ with dried leaf extract of Pongamia pinnata (L) Pierre. UV-visible spectroscopy studies were carried out to quantify the formation of silver nanoparticles. Transmission electron microscopy, X-ray diffraction and Fourier transform infrared spectroscopy were used to characterize the silver nanoparticles. TEM image divulges that silver nanoparticles are quite polydispersed, the size ranging from $20 \mathrm{~nm}$ to $50 \mathrm{~nm}$ with an average of $38 \mathrm{~nm}$. Water soluble heterocyclic compounds such as flavones were mainly responsible for the reduction and stabilization of the nanoparticles. Silver nanoparticles were effective against Escherichia coli (ATCC 8739), Staphylococcus aureus (ATCC 6538p), Pseudomonas aeruginosa (ATCC 9027) and Klebsiella pneumoniae (clinical isolate). The move towards extracellular synthesis using dried biomass appears to be cost effective, eco-friendly to the conventional methods of nanoparticles synthesis.

Keywords: Extracellular synthesis; Silver nanoparticle; Pongamia pinnata; Antibacterial

Citation: Rajesh W. Raut, Niranjan S. Kolekar, Jaya R. Lakkakula, Vijay D. Mendhulkar and Sahebrao B. Kashid, "Extracellular synthesis of silver nanoparticles using dried leaves of pongamia pinnata (L) pierre", Nano-Micro Lett. 2, 106-113 (2010). doi: 10.5101/nml.v2i2.p106-113

Nanobiotechnology, a branch of Nanoscience has been playing a decisive role in $21^{\text {st }}$ century in deciphering diverse tribulations particularly in the field of farming, medication and electronics. Nanoscience poses a basic scientific challenge as it requires a control over the connections between atoms. All physiochemical methods of nanoparticle synthesis are having inherent limitations up to a certain extent which impose an important hurdle in the maturation of this science. The improvement in the experimental procedures for controlling shape and size of nanoparticles is one of the challenges for material scientists. Equally, the development of ecofriendly cost effective methods is also in demand. Consequently, viable production of metal nanoparticles is mostly studied.

The possibility of utilizing biological materials for metal nanoparticle synthesis has appeared as the most efficient and greener approach [1]. The present decade has witnessed the rapid shift in synthesis strategies from physicochemical methods to biological agents such as bacteria, fungi and plants for nanoparticle synthesis [2-4]. The idea of employing biomaterials for nanoparticle synthesis is an extension of the well established fact that the various organisms such as diatoms, magnetostactic and S-layer bacteria are capable to synthesize nanoscale materials [5].

Both living and dead microorganisms are gaining importance by virtue of their facile assembly of nanoparticles. Prokaryotic bacteria have primarily attracted the attention in the area of biosynthesis of silver nanoparticles [6-8]. However, reduction of silver ions using these microorganisms results in the deposition of the particles in the periplasmic space and hence requires either sonication or use of permeabilizing agents 
to extract the silver nanoparticles [9]. Filamentous fungi on the other hand are capable of synthesizing the silver nanoparticles extracellularly but the downstream processing and the biomass handling make them difficult [10-12].

The biomaterial as reducing agent is a viable alternative to the current physiochemical methods which utilize intense energy, hazardous chemicals and are expensive. Furthermore, metal nanocolloids produced by the physiochemical methods tend to aggregate with time and consequently cannot be stored for longer time. Amongst biomaterials, the phytosynthetic method is a viable alternative to prokaryotes and fungi since the downstream processing and the handling of the biomass is easier. Consequently the phytosynthetic method is preferred. Bioreduction of gold and silver ions to yield metal nanoparticles using Geranium leaf broth [13], Neem leaf broth [14], lemongrass extract [15], Tamarind leaf extract [16], Aloe vera plant extracts [17] and Gliricidia sepium (Jacq.) Kunth ex Walp leaf broth [18] has been reported. The above methods have made use of the plant broth prepared by boiling finely chopped fresh leaves and some of them require addition of stabilizing agents or the use of ammonia [17] as accelerating agents. Moreover, the procedure involves continuous agitation of the broth after the addition of the salt solution.

In the present study the leaf broth prepared using dried leaf biomass of Pongamia pinnata (Linn) Pierre was used to synthesize silver nanoparticles from silver nitrate $\left(\mathrm{AgNO}_{3}\right)$. Pongamia pinnata is rich in nitrogenase and secondary metabolites which are medicinally important. Comparative experiments were carried out to explore the effect of biomass dosage on the reduction of the silver ions and uniformity of the synthesized nanoparticles. The efficacy of the synthesized nanoparticle against bacterial strains was also studied.

\section{Experiments}

\section{Preparation of dried biomass}

The twigs containing mature leaves of Pongamia pinnata

(L.) Pierre were collected from University of Mumbai campus. The mature, undamaged and disease free leaves were selected and washed thoroughly with water using Teepol and later the surface was sterilized with $0.1 \% \mathrm{HgCl}_{2}$ for $2 \sim 3$ minutes under laminar air flow. The washed leaves were kept in the oven at $40^{\circ} \mathrm{C}$ overnight for drying. The biomass used for the reduction was prepared by grinding the dried leaves followed by sieving the leaf powder using 15 mesh size sieves.

\section{Synthesis of silver nanoparticles}

In typical synthesis process, accurately weighed dried leaf biomass of Pongamia ( $0.1 \mathrm{~g}, 0.5 \mathrm{~g}$, and $1 \mathrm{~g}$ ) was added to the sterile $50 \mathrm{~mL} 1 \mathrm{mM}$ aqueous $\mathrm{AgNO}_{3}$ [Merck] solution in Dippy's jar of $250 \mathrm{~mL}$ under aseptic conditions. The jars were then incubated at room temperature under static condition.

\section{UV-Vis Spectrum analysis}

The bioreduction of silver ions was monitored by UV Visible absorption measurements at room temperature as function of time using Shimadzu UV 1700 spectrometer. The reduction of silver ions was confirmed by qualitative testing of supernatant (obtained after centrifugation) with $\mathrm{NaCl}$, no precipitation was observed. $1 \mathrm{mM} \mathrm{AgNO}_{3}$ solution was used for base line correction.

\section{X-ray Diffraction (XRD) Measurements}

The completely bioreduced sample was concentrated in concentrator (Eppendorf) at $60^{\circ} \mathrm{C}$ to reduce the volume of the reaction mixture. The concentrated solution was then centrifuged [Hermle Labortechnik (Siemensstr 2578564 Wehingen)] at $15000 \mathrm{rpm}$ for 15 minutes. The pellet obtained, was washed and redispersed in deionized water. The repeated centrifugation and redispersion in deionized water was carried out to remove the water soluble biomolecules such as proteins and secondary metabolites. XRD measurements of purified silver nanoparticle solution casted onto the glass substrate was carried out using Phillips PW 1830 instrument operating at a voltage of $40 \mathrm{kV}$ and current of $20 \mathrm{Ma}$ with $\mathrm{Cu} \mathrm{K}(\alpha)$ radiation of $1.54187 \mathrm{~nm}$ wavelength.

\section{Transmission Electron Microscopy (TEM) Measurements}

The sample purified as stated in XRD measurement section was sonicated (Vibronics VS 80) for 5 minutes. A drop of the sonicated solution was placed on carbon coated copper grid and later exposed to infrared light (30 minutes) for solvent evaporation. TEM measurements were performed on PHILIPS model CM 200 instrument operated at an accelerating voltage of $200 \mathrm{kV}$ with resolution of $0.23 \mathrm{~nm}$.

\section{Fourier Transform Infrared (FTIR) Spectroscopy Measurements}

The FTIR measurements were carried out for both the dried biomass of Pongamia pinnata leaves and washed silver nanoparticle solution, free from any biomass residue or compound except the capping ligand using Perkin Elmer (Spectrum One) spectrophotometer.

\section{Antibacterial Studies}

The antibacterial activity of silver nanoparticles was studied against Staphylococcus aureus (ATCC 6538P), Escherichia coli (ATCC 8739), Pseudomonas aeruginosa 
(ATCC 9027) and Klebsiella pneumoniae (Clinical isolate) by agar disc diffusion method [19]. The 18 hrs-revived cultures were prepared in nutrient broth [composition ( $\mathrm{gm} / \mathrm{litre})$ : peptone 15.0; yeast extract 3.0 ; sodium chloride 6.0 ; D (+) glucose 1.0].

The two replica of the respective microorganism were prepared by spreading $50 \mu \mathrm{l}$ (inoculum concentration $1.2 \times 10^{8}$ $\mathrm{CFU} / \mathrm{mL}$ ) of revived log phase culture on the nutrient agar plate [composition (gm/litre): peptone 15.0; yeast extract 3.0; sodium chloride 6.0; D (+) glucose 1.0; agar-agar 12.0] with the help of spreader. Discs of Whatman filter paper No. 40 was made having diameter of $10 \mathrm{~mm}$. The pure aliquot of plant material of $25 \mu \mathrm{l}$ was placed on the first disc as a control. $25 \mu \mathrm{l}$ sample of synthesized silver nanoparticles (prepared using $0.1 \mathrm{gm}$ dried leaves) was placed on the second disc. The third disc was used for $25 \mu \mathrm{l}$ Ampicillin (Ampilin 250 Hetero healthcare Ltd.) with concentration of $125 \mu \mathrm{g} / \mathrm{mL}$ as standard. The Petri plates were incubated in dark at $37^{\circ} \mathrm{C}$.

\section{Results and Discussion}

Development of easy, reliable and eco-friendly methods helps in endorsing extra interest in the synthesis and application of nanoparticles which are good for mankind [1]. In this context the utilization of biological systems for nanoparticle synthesis is notable alternative for the advancement of multifaceted approach. Biological systems have shown the ability to interact with metal ions and reduce them to form metallic nanoparticles [20-21].

\section{UV -Visible studies}

The relationship between the UV-visible radiation absorbance characteristics and the absorbate's size and shape is well established [13-18]. Consequently size and shape of nanoparticles in aqueous suspension can be judged by UV-visible absorbance studies. By employing the variable amount of biomass $(0.1,0.5,1.0 \mathrm{~g})$ with $1.0 \mathrm{mM} \mathrm{AgNO}_{3}$, the effect of biomass amount on rate of bioreduction and size of the target product was studied. The amount of the biomass was found to play a decisive role in size distribution of $\mathrm{Ag}$ nanoparticles. Figure (1) illustrates the absorbance spectra of reaction mixture containing aqueous silver nitrate $(1 \mathrm{mM})$ and Pongamia leaf broth (prepared from $0.1 \mathrm{~g}$ dried leaf powder) at various time intervals. The absorption spectra recorded divulge the production of $\mathrm{Ag}$ nanoparticles within $9 \mathrm{hrs}$. Upon addition of the plant broth to the solution of $\mathrm{AgNO}_{3}$, the solution changed from yellowish green to brown (see Fig. 2 (b)). The final color deepens and turns brownish with the increase of time. The intensity of the absorbance increased as the reaction proceeded. Silver nanoparticles display intense yellowish brown colour in water. This intense colour arises from the surface plasmons, which are dipole oscillation arising when an electromagnetic field in the visible range is coupled to the collective oscillations of conduction electrons [13].

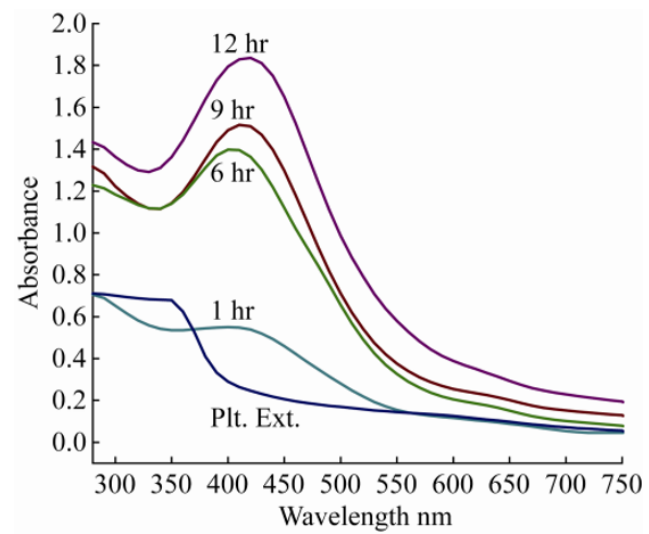

FIG. 1. UV-Vis spectra recorded at various time intervals for the reaction mixture prepared using Silver nitrate $(1 \mathrm{mM})$ and $0.1 \mathrm{~g}$ dried leaf powder of Pongamia pinnata.

It is well known that the metal nanoparticles in size ranging from 2 to $100 \mathrm{~nm}$ exhibit strong but broad surface plasmon peak. Minima at $\sim 320 \mathrm{~nm}$ observed in all the reaction mixtures correspond to the wavelength at which the real and imaginary parts of the dielectric function of silver almost vanish [17]. With the increase in the particle size, the optical absorption spectra of metal nanoparticles that are dominated by surface plasmon resonances (SPR) shift towards longer wavelengths. The position of absorption band also strongly depends upon dielectric constant of the medium and surface-adsorbed species [22]. As indicated by Mie's theory, spherical nanoparticles give rise to a single SPR band in the absorption spectra, whereas anisotropic particles confer two or more SPR bands depending on the shape of the particles [23]. In present investigation, all reaction mixtures show a single SPR band revealing spherical shape of silver nanoparticles, which was further confirmed by TEM images.

The reduction of $\mathrm{Ag}^{+}$ions occurs rapidly, completing within $9 \mathrm{hrs}$ of reaction. This was confirmed by performing qualitative analysis for the presence of free $\mathrm{Ag}^{+}$ions with $\mathrm{NaCl}$ in the supernatant obtained after centrifugation of the reaction mixture. The silver nanoparticles obtained from the reaction mixture containing $1 \mathrm{mM} \mathrm{AgNO}$ and $0.1 \mathrm{~g}$ of dried leaf powder were purified and further analyzed. The silver nanoparticles were found to be remarkably stable even after 6 months. Rapid synthesis of stable silver nanoparticles using Geranium leaf broth (20 g of leaf biomass) and $1 \mathrm{mM}$ aqueous $\mathrm{AgNO}_{3}$ have been reported by Sastry et al. [13]. Shivshankar et al. [14] 
reported rapid synthesis of stable silver, gold and bi-metallic $\mathrm{Ag} / \mathrm{Au}$ core shell nanoparticles using $20 \mathrm{~g}$ of leaf biomass of Azadiracta indica and $1 \mathrm{mM}$ aqueous $\mathrm{AgNO}_{3}$. Similarly, Pratap and coworkers [17] reported synthesis of gold and silver nanoparticles using Aloe vera leaf extract with the use of ammonia as an accelerating agent for synthesis of silver. All of

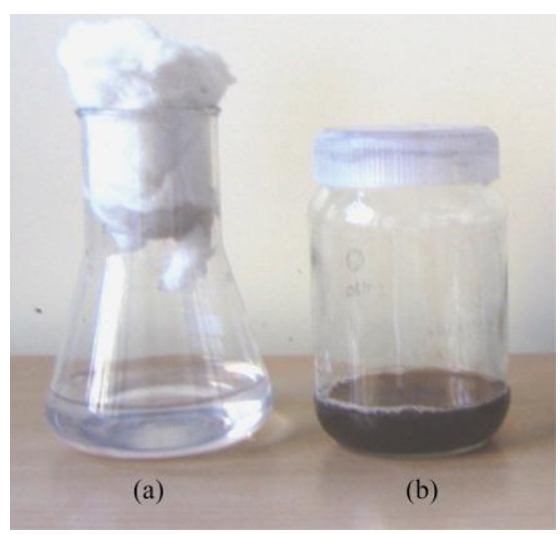

FIG. 2. Showing colour changes (a) $1 \mathrm{mM} \mathrm{AgNO}_{3}$ and (b) synthesized nanoparticles showing brownish colour.

them used leaf broth prepared by boiling finely chopped fresh leaves and the procedure involves continuous agitation of the broth after the addition of the salt solution.

The present study reports the use of oven dried leaf biomass, which is free from external stabilizing and accelerating agents and does not require continuous agitation. The reduction of the silver ions is moderately rapid at the ambient conditions. This is novel and intriguing to the material science as the studied leaf biomass has the capability to reduce metal ions at ambient conditions. Furthermore the biomass handling and processing is less stringent since it does not require boiling or subsequent treatment. Instead of sun drying the leaf biomass was oven dried at $40^{\circ} \mathrm{C}$ to avoid the possible contamination due to particulate matter. Also the sun drying process is time consuming and has geographical constraints.

\section{TEM Analysis}

TEM images which constitute large number of uniform nanoparticles revealed that the $\mathrm{Ag}$ nanoparticles produced by reduction of $\mathrm{Ag}^{+}$ions with $0.1 \mathrm{~g}$, biomass are predominantly spherical (see Fig. 3(a-c)). At low magnification (see Fig. (3c)) TEM images show large number of silver nanoparticles which are quite polydisperse and ranges in size from $20 \sim 50 \mathrm{~nm}$ with calculated average size of $38 \mathrm{~nm}$. Figure 3(b) shows clear morphology of silver nanoparticles. These nanoparticles appear to have assembled into very open, quasi-linear superstructures rather than a dense closely packed assembly [13]. The figure also reveals that nanoparticles are not in physical contact but are evenly separated. The images also clearly show an approximate $5 \mathrm{~nm}$ thickness coating around the nanoparticles which can be assigned to bioorganic compounds present in the leaf broth [13]. This is evident by number of sharp Braggs reflection observed in the XRD spectra.

\section{XRD studies}

The XRD-spectrum of purified sample of silver

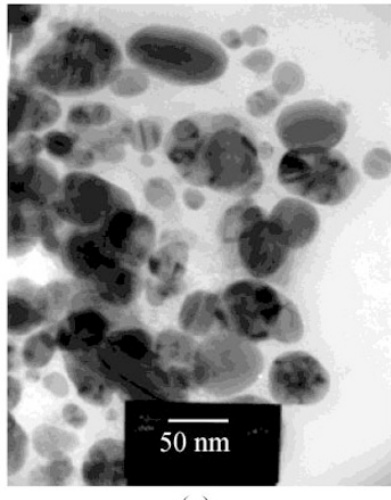

(a)

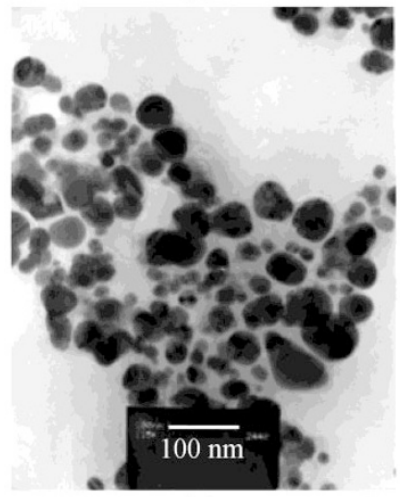

(c)

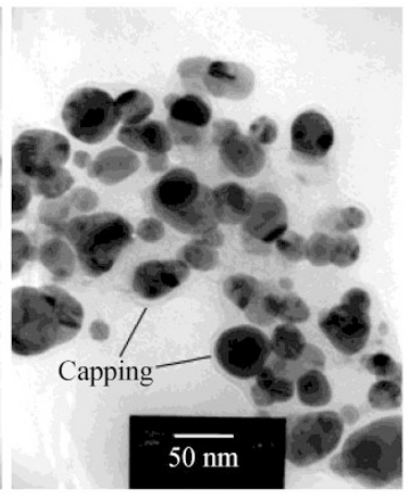

(b)

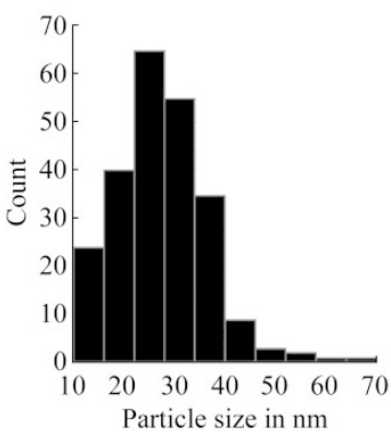

(d)
FIG. 3. TEM Images of Ag Nanoparticles (a) 2 Lakh magnification; (b) Nano particle showing capping; (c) 1 Lakh magnification; (d) Particle size distribution extracted from TEM images (235 particles counted).

nanoparticles synthesized using $0.1 \mathrm{~g}$ dried leaf biomass and $1 \mathrm{mM} \mathrm{AgNO}_{3}$ (see Fig. (4)) show a number of Braggs reflections that can be indexed on the basis of the face centered cubic structure of silver. The peaks observed in the spectrum at $2 \theta$ values of $38.06^{\circ}, 44.64^{\circ}, 64.58^{\circ}$ and $77.62^{\circ}$, corresponds to 111, 200, 220, and 311 planes for silver, respectively. This clearly indicates that the silver nanoparticles formed by the reduction of $\mathrm{Ag}^{+}$ions by the Pongamia leaf extract are crystalline in nature [7]. As mentioned in method section, the silver nanoparticles were repeatedly centrifuged and redispersed in sterile distilled water prior to XRD and TEM analysis, ruling out possibility the presence of any free compound/protein that might independently crystallize and give rise to Braggs reflections. The intense background noise is due to the protein shell around the nanoparticles [24]. An unidentified peak at $46^{\circ}$ 


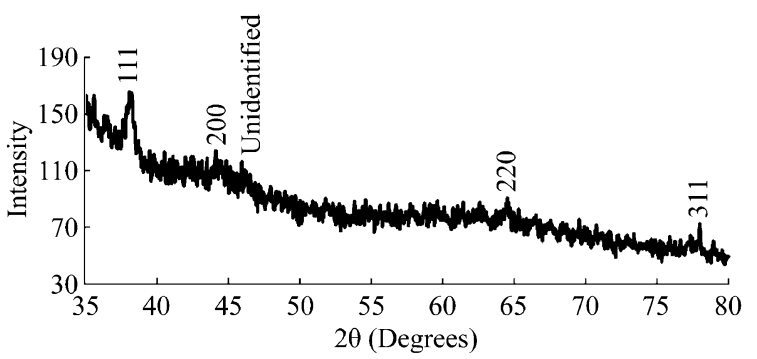

FIG. 4. XRD patterns of capped silver nanoparticles synthesized using dried leaves of $P$. pinnata.

is possibly due to crystalline nature of the capping agent [10, 13].

\section{FTIR studies}

The precise mechanism of the bioreduction is not fully understood. Mukherjee and co-workers [10] have reported that the reduction process of $\mathrm{Ag}^{+}$to $\mathrm{Ag}$ nanoparticles occurs possibly in the presence of enzyme NADPH-dependent dehydrogenase. The exact route in which the electrons are shuttled is matter of investigation. Also the information regarding environment responsible for high stability of metal nanoparticles is not comprehensively available. FTIR analysis of isolated silver nanoparticles free from proteins and water soluble compounds was done in this direction. The analysis of IR spectra gives an idea about biomolecules bearing different functionalities which are present in underlying system. Representative spectra (see Fig. (5b)) manifest major IR bands at $\sim 1651,2083,3418 \mathrm{~cm}^{-1}$, while the minor bands at $\sim 1079$ and $\sim 1538 \mathrm{~cm}^{-1}$. The FTIR spectra of dried biomass (see Fig. (5a)) show bands at $\sim 1069, \sim 1240, \sim 1314, \sim 1390, \sim 1447, \sim$ 1515, 1561, 1620, 1653, 1734, 2852, 2920, 3306, $\sim 3441$ and $\sim 3656 \mathrm{~cm}^{-1}$.

The band around $\sim 1069 \mathrm{~cm}^{-1}$ can be assigned to the ether linkages or -C-O- [14,25], whereas, the band around $\sim 1314$ $\mathrm{cm}^{-1}$ and $1390 \mathrm{~cm}^{-1}$ can be assigned to geminal methyls [14] (see Fig. (5a)). To a large extent, the band at $\sim 1069 \mathrm{~cm}^{-1}$ might be contributed by the -C-O- groups of the polyols such as flavones, terpenoids and the polysaccharides present in the biomass [25]. The absorbance band centered at $\sim 1620$ and $1653 \mathrm{~cm}^{-1}(5 \mathrm{a})$ is associated with the stretching vibration of $-\mathrm{C}=\mathrm{C}$ - or aromatic groups [14,25]. The band around $\sim 1734$ can be assigned to $\mathrm{C}=\mathrm{O}$ stretching vibrations of the carbonyl functional group in ketones, aldehydes, and carboxylic acids $[13,17,25]$. Besides, the spectrum (5a) also exhibits two intense bands at $\sim 2852$ and $2920 \mathrm{~cm}^{-1}$, respectively assigned to the symmetric and asymmetric stretching vibration of $\mathrm{sp} 3$ hybridized $-\mathrm{CH}_{2}$ groups that are not observed in the spectrum (5b) of isolated silver nanoparticles, suggesting that $-\mathrm{CH}_{2}$

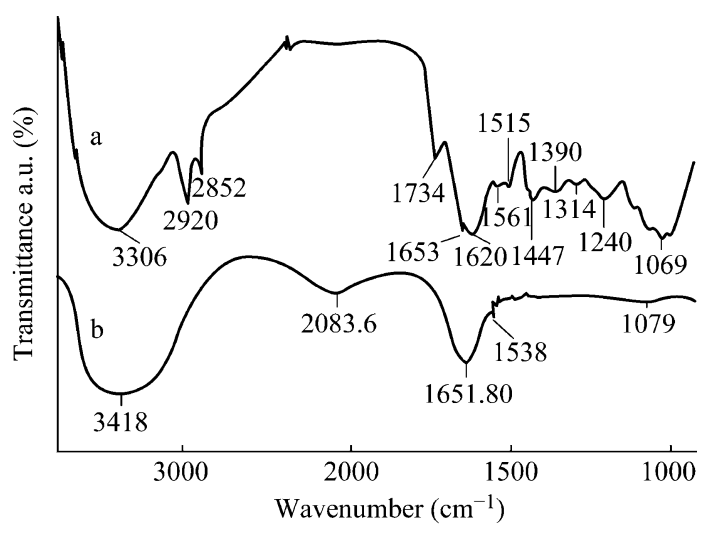

FIG. 5. FTIR spectra of (a) dried leaves of Pongamia pinnata; (b) capped silver nanoparticles.

groups are probably absent in solution [10].

The band at $\sim 1651 \mathrm{~cm}^{-1}$ (5b) is due to amide I bond of proteins, indicating predominant surface capping species having $-\mathrm{C}=\mathrm{O}$ functionality which are mainly responsible for stabilization. However, albeit there is adequate evidence for the presence of surface bound proteins, the conclusion regarding the presence of the specific compound cannot be made unless the individual component is isolated and identified. Furthermore, an interfacial interaction of these biomolecules with core particles is the matter of investigation. Sastry and co-workers [16] have reported that the species having dipole moment such as acetone binds strongly and reversibly to the gold nanoparticles in vapor phase. This supports the strong binding of $-\mathrm{C}=\mathrm{O}$ functionality from medium, to the core particles. A broad intense band at $\sim 3400 \mathrm{~cm}^{-1}$ in both the spectra can be assigned to the N-H stretching frequency arising from the peptide linkages present in the proteins of the extract [10]. The shoulders around the band can be identified as the overtone of the amide-II band and the stretching frequency of the O-H band, possibly arising from the carbohydrates and/or proteins present in the sample. The flattening of the shoulders in Fig. (5b) indicates decrease in the concentration of the peptide linkages in the solution [10]. The spectra (see Fig. (5b)) also exhibit broad asymmetric band at $\sim 2100 \mathrm{~cm}^{-1}$ that can be assigned to the N-H stretching band in the free amino groups of silver nanoparticles.

The bands of functional groups such as -C-O-C- , -C-Oand $-\mathrm{C}=\mathrm{O}$ are derived from the heterocyclic water soluble compounds present in the dried biomass, which as seen in the IR spectra of dried biomass is in good agreement with the value reported in the literature. Since these band disappeared in the IR spectra of the final product it can be concluded that the reducing agents are largely derived from these water soluble heterocyclic compounds. Yin et al [26-27] has reported the 
presence of prenylated flavonoid derivatives pongaflavanol and tunicatachalcone in Pongamia pinnata, where the latter one is proposed as biogenetic precursor of the former one and the reaction is effected by flavanone/dihydroflavanonol NADPH-dependent reductase. Therefore, it reflects that water soluble heterocyclic compounds such as flavones are the reducing and capping ligands of the nanoparticles.

In the polyol synthesis, gold nanoparticles are extensively studied for, and both oxygen and nitrogen atoms of pyrrolidone unit can facilitate the adsorption of PVP on to the surface of metal nanostructures to fulfill the protection of nanoparticles [28]. Likewise, the oxygen atoms herein might facilitate the adsorption of the heterocyclic components on to the particle surface in stabilizing the nanoparticles. It is also evident from the differences in the peak for dried powder and silver nanoparticles that the flavones are responsible for the bioreduction. Flavones could be adsorbed on the surface of the metal nanoparticles, possibly by interaction through $\pi$-electrons of carbonyl groups in the absence of other strong ligating agents in sufficient concentrations [14]. On comparing Fig. (5a) and (5b) it can be outlined that the disappearance of the band around $1069 \mathrm{~cm}^{-1}$ shows that the polyols are mainly responsible for the reduction of silver ions.

\section{Antibacterial Studies}

Antibacterial activity of biogenic silver nanoparticles was evaluated by using standard zone of inhibition (ZOI) microbiology assay. The nanoparticles showed inhibition zone against all the studied bacteria (see Table (1)), (see Fig. (6)). Maximum zone of inhibition was found to be $12 \mathrm{~mm}$ for Staphylococcus aureus ATCC 6538P and Klebsiella pneumoniae (Clinical isolate) and minimum of $8 \mathrm{~mm}$ for $E$. coli.

Silver nitrate which is readily soluble in water has been exploited as an antiseptic agent for many decades [29]. Dilute solution of silver nitrate has been used since the 19th century to treat infections and burns [30]. The exact mechanism of the antibacterial effect of silver ions is partially understood. Literature survey reveals that the positive charge on the Ag ion is crucial for its antimicrobial activity. The antibacterial activity is probably derived, through the electrostatic attraction between negative charged cell membrane of microorganism and positive charged nanoparticles [31-33]. However, Sondi and Salopek-Sondi [34] reported that the antimicrobial activity of silver nanoparticles on Gram-negative bacteria was dependent on the concentration of $\mathrm{Ag}$ nanoparticles and was closely associated with the formation of pits in the cell wall of bacteria. Accumulation of the Ag nanoparticles in the pits results in the permeability of the cell membrane, causing cell death. Similarly, Amro et al [35] suggested that depletion of the silver metal from the outer membrane may cause progressive release of lipopolysaccharide molecules and membrane proteins. This results in the formation of irregularly shaped pits and hence increases the membrane permeability. Similar mechanism has been reported to be operative by Sondi and Salopek-Sondi [34] in the membrane structure of E. coli during treatment with $\mathrm{Ag}$ nanoparticles. Recently, Kim and co-workers [36] have reported that the silver nanoparticles generate free radicals that are responsible for damaging the membrane. They also speculated that the free radicals are developed from the surface of the silver nanoparticles. Lee et al [37] investigated the antibacterial effect of nanosized silver colloidal solution against $S$. aureus and $K$. pneumoniae after padding the solution on textile fabrics. Shrivastava et al [38] studied antibacterial activity against $E$. coli (ampicillin resistant), E. coli, S. aureus, and $S$. typhi (multi-drug resistant). They reported that the effect was dose dependent and was more pronounced against gram-negative organisms than gram-positive ones. They found that the major mechanism through which silver nanoparticles manifest antibacterial properties was either by anchoring or penetrating the bacterial cell wall, and modulating cellular signaling by dephosphorylating putative key peptide substrates on tyrosine residues [38]. Similarly, Chun-Nam and coworkers [39] reported that the silver nanoparticles target the bacterial

Table 1. Antibacterial activity of silver nanoparticles.

\begin{tabular}{|c|c|c|c|c|}
\hline \multirow{2}{*}{ Sr. No. } & \multirow{2}{*}{ Name of the Strain } & \multicolumn{3}{|c|}{ Diameter of Zone of Inhibition in $\mathrm{mm}$} \\
\hline & & Ampicillin & $\mathrm{AgNO}_{3}$ & Silver Nanoparticles \\
\hline 1 & Pseudomonas aeruginosa (ATCC 9027) & 15 & 7 & 10 \\
\hline 2 & Escherichia coli (ATCC 8739) & 20 & 6 & 8 \\
\hline 3 & Klebsiella pneumoniae (Clinical isolate) & 16 & 7 & 12 \\
\hline 4 & Staphylococcus aureus (ATCC 6538P) & 20 & 8 & 12 \\
\hline
\end{tabular}


membrane, leading to a dissipation of the proton motive force resulting in the collapse of the membrane potential. They also proposed that the silver nanoparticles mediated antibacterial effects in a much more efficient physiochemical manner than $\mathrm{Ag}^{+}$ions. The antibacterial efficacy of the biogenic silver nanoparticles reported in the present study may be ascribed to the mechanism described above but it still remains to clarify the exact effect of the nanoparticles on important cellular metabolism like DNA, RNA and protein synthesis.

\section{Conclusion}

The method represents an example of clean, nontoxic and ecofriendly method for obtaining silver nanoparticles. The capping around each particle furnish uniform chemical environment formed by the bioorganic compound present in the leaf broth, which may be mainly responsible for the stabilization of the particles. This method provides a simple and efficient route for the synthesis of nanoparticles with tunable optical properties directed by particle size. From the point of view of nanotechnology this is a significant advancement to synthesize silver nanoparticles economically.

The silver nanoparticles show distinct polydispersity as it shows particle size between the ranges of 20 to $50 \mathrm{~nm}$ with an average size of $38 \mathrm{~nm}$. The maximum nanoparticles showed particle size of $30 \mathrm{~nm}$, distinct cap was observed around the nanoparticles, which is due to the flavonoids present in the leaf broth of Pongamia pinnata and is mainly responsible for the reduction of silver ions. The identification of capping agents and tapping the enzymes responsible for the reduction process may open up new avenues in the field of nanoparticle stabilization.

Investigation on the antibacterial effect of nanosized silver colloidal solution against $S$. aureus (ATCC 6538), $K$. pneumoniae, P. aeruginosa (ATCC9 027) and E. coli (ATCC 8739) reveals high efficacy of silver nanoparticles as a strong antibacterial agent. This can be useful in food industries, cosmetic industries and medicines. The important question regarding biocompatibility of nanoparticles is yet to be answered.

We sincerely acknowledge the facilities provided by The Institute of Science and Tata Institute of Fundamental Research, Mumbai.

Received 4 March 2010; accepted 30 March 2010; published online 20 May 2010.

\section{References}

1. D. Bhattacharya and R. K. Gupta, Crit. Rev. Biotechnol. 25, 199 (2005). doi:10.1080/07388550500361994.

2. K. Prasad, K. J. Anal and A. R. Kulkarni, Nanoscale Res. Lett. 2, 248 (2007). doi:10.1007/s11671-007-9060-x.

3. P. Mukherjee, S. Senapat, D. Mandal, A. Ahmad, M. I. Khan, R. Kumar and M. Sastry, Angew. Chem., Int. Ed. Engl. 40, $3585 \quad$ (2001a). $\quad \underline{\text { doi:10.1002/1521-3773 }}$ (20011001)40:19<3585::AID-ANIE3585>3.0.CO;2-K.

4. G. Singaravelu, J. S. Arockiamary, V. Ganesh Kumar and K. Govindaraju, Colloids Surf. B. Biointerf. 57, 97 (2007). doi:10.1016/j.colsurfb.2007.01.010.

5. D. Pum and U. B. Sleytr, Trends Biotechnol. 17, 8 (1999). doi:10.1016/S0167-7799(98)01221-9.

6. T. Klaus, R. Joerger, E. Olsson and C. G. Granqvist, Trends Biotechnol. 19, 15 (2001). doi:10.1016/S01677799(00)01514-6.

7. R. Joerger, T. Klau and C. G. Granqvist, Adv. Mater. 12, 407 (2000). doi:10.1002/(SICI)1521-4095(200003)12:6< 407::AID-ADMA407>3.0.CO;2-O

8. B. Nair and T. Pradeep, Cryst. Growth Des. 2, 293 (2002). doi: $10.1021 / \operatorname{cg} 0255164$.

9. D. Mandal, M. E. Bolander, D. Mukhopadhyay, G. Sarkar and P. Mukherjee, Appl. Microbiol. Biotechnol. 69, 485 (2006). doi:10.1007/s00253-005-0179-3.

10. P. Mukherjee, M. Roy, B. P. Mandal, G. K. Dey, P. K. Mukherjee, J. Ghatak, A. K. Tyagi and S. P. Kale, Nanotechnology 19075103 (2008).

11. A. Ingle, A. Gade, S. Pierrat, C. Sönnichsen and M. Rai, Curr. Nanosci. 4, 141 (2008). doi:10.2174/1573413 08784340804.

12. K. Bhainsa and S. D'Souza, Colloid Surf. B 47, 160 (2006).

13. S. Shiv Shankar, A. Ahmad and M. Sastry, Biotechnol. Prog. 19, 1627 (2003). doi:10.1021/bp034070w.

14. S. Shiv Shankar, A. Rai, A. Ahmad and M. Sastry, J. Colloid Interf. Sci. 275, 496 (2004).

15. S. Shiv Shankar, A. Rai, A. Ahmad and M. Sastry, Chem. Mater. 17, 566 (2005). doi:10.1021/cm048292g.

16. B. Ankamwar, M. Chaudhary and M. Sastry, Synth. React. Inorg. Metal-Org. Nano. Metal Chem. 35, 19 (2005).

17. S. C. Prathap, M. Chaudhary, R. Pasricha, A. Ahmad and M. Sastry, Biotechnol. Prog. 22, 577 (2006). doi:10.1021/ bp0501423. 
18. R. W. Raut, J. R. Lakkakula, N. S. Kolekar, V. D. Mendhulkar and S. B. Kashid, Curr. Nanaosci. 5, 117 (2009). doi:10.2174/157341309787314674.

19. E. Rodrigues, S. Tilvi and C. G. Naik, J. Exp. Mar. Biol. Ecol. 309, 121 (2004). doi:10.1016/j.jembe.2004.03.010.

20. T. J. Beveridge and R. G. E. Murray, J. Bacteriol. 141, 876 (1980).

21. T. J. Beveridge, M. N. Hughes, H. Lee, K. T. Leung, R. K. Poole, I. Savvaidis, S. Silver and J. T. Trevors, Adv. Microb. Physiol. 38, 178 (1997).

22. Y. Xia and N. J. Halas, Mrs. Bull. 30, 338 (2005).

23. G. Mie, Ann. D. Physik. 25, 377 (1908). doi:10.1002/andp. 19083300302.

24. N. Vigneshwaran, N. M. Ashtaputre, P. V. Varadarajan, R. P. Nachane, K. M. Paralikar and R. H. Balasubramanya, Mater. Lett. 61, 1413 (2007). doi:10.1016/j.matlet. 2006.07.042.

25. J. Huang, Q. Li, D. Sun, Y. Lu, Y. Su, X. Yang, H. Wang, Y. Wang, W. Shao, N. He, J. Hong and C. Chen, Nanotechnology 18, 1 (2007).

26. H. Yin, S. Zhang, J. Wu, H. Nan, L. Long, J. Yang and Q. Li, Molecules 11, 786 (2006). doi:10.3390/11100786.

27. H. Yin, S. Zhang and J. Wu, Z. Naturforsch 60b, 356 (2005).

28. Y. Xiong, I. Washio, J. Chen, H. Cai, Z. Y. Li and Y. Xia, Langmuir 22, 8563 (2006). doi:10.1021/1a061323x.

29. A. B. Lansdown, J. Wound Care 11, 125 (2002a).

30. C. L. Fox Jr., Arch. Surg. 96, 184 (1968).
31. T. Hamouda, A. Myc, B. Donovan, A. Shih, J. D. Reuter and J. R. Baker Jr, Microbiol. Res. 156, 1 (2000). doi:10.1078/0944-5013-00069.

32. P. Dibrov, J. Dzioba, K. K. Gosink and C. C. Hase, Antimicrob Agents Chemother. 46, 2668 (2002). doi:10.1128/AAC.46.8.2668-2670.2002.

33. I. Dragieva, S. Stoeva, P. Stoimenov, E. Pavlikianov and K. Klabunde, Nanostruct. Mater. 12, 267 (1999). doi:10.1016/S0965-9773(99)00114-2.

34. I. Sondi and B. Salopek-Sondi, J. Colloid Interf. Sci. 275, 177 (2004). doi:10.1016/j.jcis.2004.02.012.

35. N. A. Amro, L. P. Kotra, K. Wadu-Mesthrige, A. Bulychev, S. Mobashery and G. Liu, Langmuir 16, 2789 (2000). doi:10.1021/la991013x.

36. J. S. Kim, E. Kuk, K. N. Yu, J. H. Kim, S. J. Park, H. J. Lee, S. H. Kim, Y. K. Park, Y. H. Park, C. Y. Hwang, Y. K. Kim, Y. S. Lee, D. H. Jeong and M. H. Cho, Nanomedicine: Nanotechnol Biol. Med. 3, 95 (2007). doi:10.1016/j.nano.2006.12.001.

37. H. J. Lee, S. Y. Yeo and S. H. Jeong, J. Mater. Sci. 38, 2199 (2003). doi:10.1023/A:1023736416361.

38. S. Shrivastava, T. Bera, A. Roy, G. Singh, P. Ramachandrarao and D. Dash, Nanotechnology 18, 225103 (2007). doi:10.1088/0957-4484/18/22/225103.

39. L. Chun-Nam, H. Chi-Ming, C. Rong, He. Qing-Yu, Y. Wing-Yiu, S. Hongzhe, T. Paul Kwong-Hang, C. Jen-Fu and C. Chi-Ming, J. Proteome Res. 5, 916 (2006). 